\title{
Determination of Important Nuclear Fragmentation Processes for Human Space Radiation Protection
}

\author{
Zi-Wei Lin \\ Mail Stop VP62, NSSTC, 320 Sparkman Drive, Huntsville, AL 35805
}

\begin{abstract}
We present a semi-analytical method to determine which partial cross sections of nuclear fragmentations most affect the shielded dose equivalent due to exposure to galactic cosmic rays. The cross sections thus determined will require more theoretical and/or experimental studies in order for us to better predict, reduce and mitigate the radiation exposure in human space explorations.
\end{abstract}

PACS numbers: $87.50 .-\mathrm{a}, 25.70 . \mathrm{Mn}, 98.70 . \mathrm{Sa}$

\section{INTRODUCTION}

Space radiation poses a serious risk to the health of astronauts in space explorations [1]. Space radiation particles mainly consist of the galactic cosmic rays (GCR) and solar energetic particles (SEP). SEPs can have a very large flux in solar particle events such as coronal mass ejections but their kinetic energies are typically below a few hundred $\mathrm{MeV}$. GCR particles are fully-ionized nuclei at much higher average energies, thus they can penetrate thick materials and pose serious health hazards, especially for long-term human space explorations. Physical and biological effects of space radiation are currently being studied [2] in order to provide astronauts enough protection against space radiation.

Reliable space radiation transport codes are essential for the prediction of radiation environments behind shielding and effective reductions of the radiation risk. A key input to these transport codes is the physics of the interactions between radiation particles and different materials. Atomic interactions which cause the ionization energy loss are well known. However, significant uncertainties exist in our understanding of the nuclear fragmentation processes of different ions. To improve the accuracy of the space radiation transport, laboratory experiments have been performed to measure fragments from the fragmentation of different elemental projectiles, e.g., at the NASA Space Radiation Laboratory at the Brookhaven National Laboratory. Because space radiation particles involve many different ions and they fragment inside materials, an important question is what partial cross sections affect the space radiation risk more and thus need to be better studied.

Here we present a semi-analytical method in order to understand the qualitative features of the effects of nuclear fragmentations on space radiation risks from GCR. In Sec.II we describe the semi-analytical method and the theoretical results obtained in the thin-shielding limit, including a list of the top 30 most important partial cross sections for both water and aluminum shielding for the 1977 solar minimum GCR environment. Sec.III shows our results for thick shielding. In Sec.IV we discuss the important effect of the unitarity relation due to the baryon number conservation, and provide two simple examples on alternate implementation of the unitarity re- lation. The section also shows the difference in the sensitivities between solar maximum and minimum GCR environments. We summarize and present our conclusions in Sec.V.

\section{THIN-SHIELDING RESULTS FROM THE SEMI-ANALYTICAL METHOD}

In the straight-ahead approximation, where fragmentation products are assumed to keep the same velocity as the projectile, the propagation equation is given by [3]

$$
\frac{\partial J_{k}(E, x)}{\partial x}=-\frac{J_{k}(E, x)}{\Lambda_{k}(E)}+\sum_{j} \frac{J_{j}(E, x)}{\Lambda_{k j}(E)}+\frac{\partial\left[\omega_{k}(E) J_{k}(E, x)\right]}{\partial E},(1)
$$

where $J_{k}(E, x)$ represents the flux of particle species $k$ with energy $E$ per nucleon at depth $x$ in a material, and $\omega_{k}(E)$ represents the ionization energy loss. The term

$$
\Lambda_{k}(E)=\frac{1}{n \sigma_{k}(E)}
$$

represents the mean-free-path of particle species $k$ in the shielding material with $n$ being the number density of nuclei in the material and $\sigma_{k}(E)$ the total inelastic cross section of particle species $k$. In the term

$$
\Lambda_{k j}(E)=\frac{1}{n \sigma_{k j}(E)}
$$

$\sigma_{k j}(E)$ represents the partial cross section for projectile $j$ to produce fragment $k$. In the thin-shielding limit, i.e., $x \rightarrow 0$, we obtain the following results to first order in $x$ :

$$
\begin{aligned}
J_{k}(E, x) \approx & J_{k}(E, 0)\left[1+\omega_{k}^{\prime}(E) x+\frac{J_{k}^{\prime}(E, 0)}{J_{k}(E, 0)} \omega_{k}(E) x\right. \\
& \left.-\frac{x}{\Lambda_{k}(E)}\right]+\sum_{j} \frac{J_{j}(E, 0)}{\Lambda_{k j}(E)} x
\end{aligned}
$$

where $\omega_{k}^{\prime}(E)$ and $J_{k}^{\prime}(E, 0)$ represent the first derivative with respect to energy $E$. Only the last two terms on the right-hand side of Eq. (4) involve the fragmentation cross sections and they do not involve the ionization energy loss $\omega_{k}(E)$. Therefore, to first order in $x$, the energy loss in 
materials does not affect the sensitivity of the shielded radiation fluxes to fragmentation cross sections.

Dose equivalent $H$ is used in this study to relate the energy spectra of radiation particles to the radiation risk:

$$
H \equiv \int Q(L) D_{L} d L
$$

where $D_{L}$ represents the distribution of absorbed dose as a function of $L$, the unrestricted Linear-Energy-Transfer (LET). The International Council on Radiation Protection (ICRP) has defined a quality factor, $Q(L)$, which roughly summarizes the data on Relative Biological Effectiveness (RBE) of different types of radiation relative to $\mathrm{X}$-ray or $\gamma$-ray radiation. Because $\mathrm{RBE}$ depends on the biological end-points, and certain types of radiation may have qualitatively different effects than X-ray or $\gamma$-ray radiation, there are significant uncertainties associated with the quality factor. In this study we use the quality factor defined in the most recent ICRP60 [4]. The dose equivalent inside soft tissue, which is simulated by water, behind depth $x$ of a shielding material is then given by

$$
H(x)=\frac{1}{\rho_{T}} \sum_{k} \int J_{k}(E, x) L_{k}(E) Q\left(L_{k}(E)\right) d E,
$$

where $\rho_{T}$ represents the density of water and $L_{k}(E)$ represents the LET value in water for particle $k$ at energy $E$ per nucleon.

Eqs. (4) and (6) lead to

$\delta H(x)=\frac{n x}{\rho_{T}} \sum_{j} \int J_{j}\left[-L_{j} Q\left(L_{j}\right) \delta \sigma_{j}+\sum_{k} L_{k} Q\left(L_{k}\right) \delta \sigma_{k j}\right] d E,(7)$

which relates the uncertainty in the dose equivalent to the uncertainty in each partial fragmentation cross section.

The baryon number conservation relates the total inelastic cross section to the partial fragmentation cross sections. When the energy of the projectile is below the threshold for baryon-antibaryon productions, about $6 \mathrm{GeV}$ per nucleon in the laboratory frame, the baryon number conservation is expressed as the following unitarity relation:

$$
A_{j} \sigma_{j}(E)=\sum_{k} A_{k} \sigma_{k j}(E)
$$

assuming the straight-ahead approximation, where fragments of the projectile can be separated from fragments of the target material. The uncertainties in the total inelastic cross section and the partial fragmentation cross sections are then related via the unitarity relation as

$$
A_{j} \delta \sigma_{j}(E)=\sum_{k} A_{k} \delta \sigma_{k j}(E)
$$

Therefore not all uncertainties in Eq. (7) are independent. Because GCR particles with kinetic energies from about $0.2 \mathrm{GeV}$ to a few $\mathrm{GeV}$ per nucleon, i.e., mostly

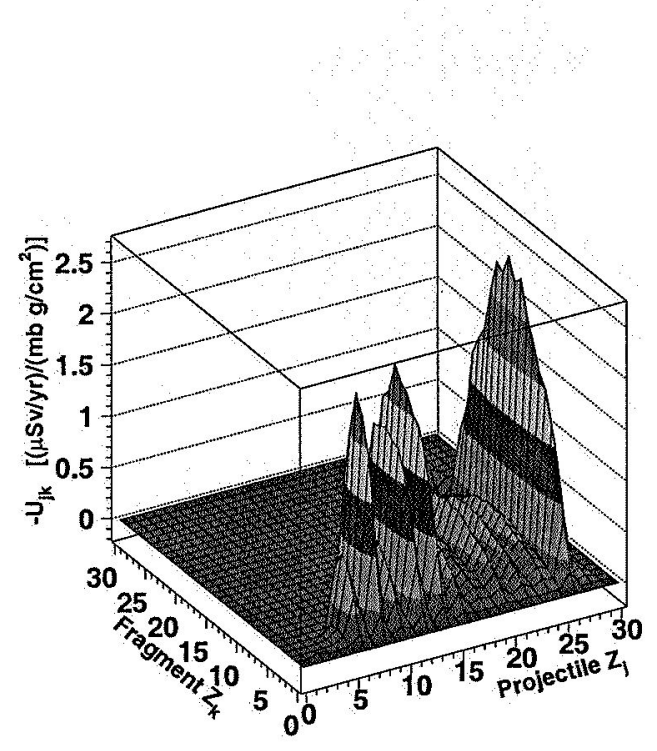

FIG. 1: (Color online) Matrix elements for the sensitivity of dose equivalent in water shielding to a given change in each partial cross section $\sigma_{k j}$ for the 1977 solar minimum GCR environment in the thin-shielding limit.

below the threshold for baryon-antibaryon productions, affect the shielded radiation exposure the most [5-7], Eq. (9) is a good approximation.

To study the sensitivity of the shielded dose equivalent to fragmentation cross sections, we need first to evaluate the change in the dose equivalent caused by the change in a partial cross section while keeping all other partial cross sections fixed. Therefore, the unitarity constraint of Eq. (9) requires us to change the total inelastic cross section accordingly, and Eq. (7) becomes

$$
\delta H(x)=\frac{n x}{\rho_{T}} \sum_{j, k} \int J_{j}\left[-L_{j} Q\left(L_{j}\right) \frac{A_{k}}{A_{j}}+L_{k} Q\left(L_{k}\right)\right] \delta \sigma_{k j} d E .(10)
$$

For energy-independent changes in the partial cross sections, $\delta \sigma_{k j}, \mathrm{Eq}$. (10) reduces to

$$
\begin{aligned}
& \delta H(x) \equiv \rho x \sum_{j, k} U_{j k} \delta \sigma_{k j} \\
& U_{j k}=\frac{n}{\rho_{T} \rho} \int J_{j}\left[-Z_{j}^{2} Q\left(Z_{j}^{2} L_{1}\right) \frac{A_{k}}{A_{j}}+Z_{k}^{2} Q\left(Z_{k}^{2} L_{1}\right)\right] L_{1} d E
\end{aligned}
$$

where $\rho$ represents the density of the shielding material, and the relation $L_{k}(E) \simeq Z_{k}^{2} L_{1}(E)$ has been used for the LET spectra with $Z_{k}$ being the charge of particle species $k$. The above matrix element $U_{j k}$ represents the sensitivity of the dose equivalent behind a material of areal density $\rho x$ to the uncertainty in the partial fragmentation cross section $\sigma_{k j}$. Note that water shielding and the 1977 solar minimum GCR environment for elements from protons to nickel in the energy range between 0.1 $\mathrm{MeV}$ per nucleon to $1000 \mathrm{GeV}$ per nucleon are used in 
the calculations unless specified otherwise, and the GCR environments in this study are taken from Ref. [8].

Fig. 1 shows the matrix elements $U_{j k}$ as a function of projectile and fragment charges. It is evident from Fig. 1 that $\mathrm{Fe}, \mathrm{Si}, \mathrm{Mg}$ and $\mathrm{O}$ projectiles contribute to the highest peaks. For a given projectile, the matrix element almost vanishes for either light or heavy (i.e., with charges near that of the projectile) fragments while peaking for medium-sized fragments. Indeed Eq. (10) shows that the contribution from projectile $j$ to $\delta H(x)$ vanishes at both the light-fragment limit $\left(A_{k} \rightarrow 0\right.$ and $\left.Z_{k} \rightarrow 0\right)$ and the heavy-fragment limit $(k \rightarrow j)$. In a simplified case where fragments have the same quality factor and the $A / Z$ ratio as the projectile, we have

$$
U_{j k} \propto-Z_{j}^{2} \frac{A_{k}}{A_{j}}+Z_{k}^{2} \simeq Z_{k}\left(-Z_{j}+Z_{k}\right)
$$

therefore the matrix elements for a given projectile $j$ would peak in the middle (at $Z_{k} \simeq Z_{j} / 2$ ). We can clearly see this feature in the shapes of the fragment distributions shown in Fig. 1

It is often more useful to evaluate the sensitivity to relative changes in the partial cross sections. Considering energy-independent relative changes in the partial cross sections, $\delta \sigma_{k j} / \sigma_{k j}$, Eq. (12) reduces to

$$
\begin{aligned}
& \delta H(x) \equiv \rho x \sum_{j, k} S_{j k} \frac{\delta \sigma_{k j}}{\sigma_{k j}}, \\
& S_{j k}=\frac{n}{\rho T \rho} \int J_{j}\left[-Z_{j}^{2} Q\left(Z_{j}^{2} L_{1}\right) \frac{A_{k}}{A_{j}}+Z_{k}^{2} Q\left(Z_{k}^{2} L_{1}\right)\right] L_{1} \sigma_{k j} d E .
\end{aligned}
$$

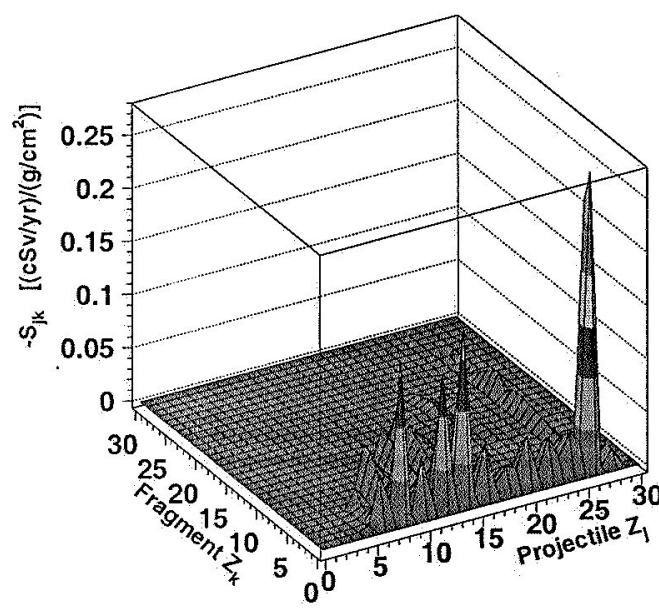

FIG. 2: (Color online) Matrix elements for the sensitivity of dose equivalent in water shielding to a given relative change in each partial cross section $\left(\delta \sigma_{k j} / \sigma_{k j}\right)$ for the 1977 solar minimum GCR environment in the thin-shielding limit.

These results are semi-analytical because they require the input of the quality factors and fragmentation cross sections. Unlike $U_{j k}$ of Eq. (12), the shapes of $S_{j k}$ depend on the material due to the $\sigma_{k j}$ term which represents fragmentations in the material. Fig. 2 shows the sensitivity matrix elements $S_{j k}$ with water as the shielding material, where values of the partial cross sections $\sigma_{k j}(E)$ are taken from Ref. [9]. We see that fragments with $Z=1$ (protons) have the largest effect on the uncertainty of the dose equivalent behind shielding, followed by fragments with $Z=2$ (alpha particles). In the same simplified case as for Eq. (13), where fragments have the same quality factor and the $A / Z$ ratio as the projectile, we have

$$
S_{j k} \propto\left(-Z_{j}^{2} \frac{A_{k}}{A_{j}}+Z_{k}^{2}\right) \sigma_{k j} \simeq Z_{k}\left(-Z_{j}+Z_{k}\right) \sigma_{k j} .
$$

Considering $\mathrm{Fe}$ projectiles for example, the factor $Z_{k}\left(-Z_{j}+Z_{k}\right)$ above is the same for fragments with $Z_{k}=1$ (protons) or fragments with $Z_{k}=25(\mathrm{Mn})$, but $\sigma_{\mathrm{pFe}} \gg \sigma_{\mathrm{MnFe}}$. For an Fe projectile at $1.2 \mathrm{GeV}$ per nucleon in water shielding, the partial contribution to the total inelastic cross section, represented by $A_{k} \sigma_{k j} /\left(A_{j} \sigma_{j}\right)$, is about $13.9 \%, 10.8 \%, 8.5 \%, 0.3 \%, 0.3 \%$, $1.1 \%, 8.8 \%$, and $11.9 \%$ for neutrons, protons, alpha particles, $\mathrm{Li}, \mathrm{O}, \mathrm{Si}, \mathrm{Cr}$ and $\mathrm{Mn}$ fragments, respectively [9]. Thus the cross section to produce a proton fragment, $\sigma_{\mathrm{pFe}}$, is about 50 times the value of $\sigma_{\mathrm{MnFe}}$. Therefore, light fragments contribute to the highest peaks in Fig. 2 partly because of the large partial cross sections to produce them.

Table I lists the top 30 most important cross sections for both water and aluminum shielding, ranked according to the absolute values of the corresponding matrix elements as given by Eq. (15). We see that the most important partial cross sections are for proton and alpha particle productions [10] from heavy projectiles such as Fe and $\mathrm{Si}$. We also find that in aluminum, another typical benchmark material for radiation evaluations, the most important cross sections are very similar to those in water shielding. The absolute values of the matrix elements in aluminum are smaller in general than those in water shielding, consistent with the fact that aluminum is less effective than water as a radiation shielding material against GCR.

Fig. 3 shows one-dimensional reductions of the above two-dimensional matrix $S_{j k}$ to both the projectile and the fragment charges. A straightforward summation of $S_{j k}$ over the fragment or the projectile charge would not be appropriate because the matrix elements, as well as the uncertainties in the partial cross sections, have different signs in general. In a simplified case where the uncertainties in the partial cross sections have the same magnitude $\left(\left|\delta \sigma_{k j}\right| / \sigma_{k j} \equiv \epsilon\right)$ but random signs, Eq. (14) reduces to

$$
\delta H(x) \approx \rho x \epsilon \sqrt{\sum_{j, k} S_{j k}^{2}}
$$

Therefore we show $\sqrt{\sum_{j} S_{j k}^{2}}$ as a function of the frag- 
TABLE I: The top 30 matrix elements in the unit of $\left[(\mathrm{cSv} / \mathrm{yr}) /\left(\mathrm{g} / \mathrm{cm}^{2}\right)\right]$ ranked according to their absolute values for the 1977 solar minimum GCR environment.

\begin{tabular}{|c|c|c|c|c|c|}
\hline \multicolumn{3}{|c|}{ For water shielding } & \multicolumn{3}{|c|}{ For aluminum shielding } \\
\hline j & $\mathrm{k}$ & $-S_{j k}$ & $\mathrm{j}$ & $\mathrm{k}$ & $-S_{j k}$ \\
\hline 26 & 1 & 0.2800 & 26 & 1 & 0.2204 \\
\hline 26 & 2 & 0.2488 & 26 & 2 & 0.1691 \\
\hline 14 & 1 & 0.1639 & 14 & 1 & 0.1173 \\
\hline 8 & 1 & 0.1495 & 8 & 1 & 0.1019 \\
\hline 12 & 1 & 0.1280 & 12 & 1 & 0.0883 \\
\hline 14 & 2 & 0.0982 & 14 & 2 & 0.0634 \\
\hline 12 & 2 & 0.0647 & 16 & 1 & 0.0395 \\
\hline 8 & 2 & 0.0627 & 12 & 2 & 0.0388 \\
\hline 10 & 1 & 0.0545 & 10 & 1 & 0.0380 \\
\hline 16 & 1 & 0.0507 & 20 & 1 & 0.0375 \\
\hline 6 & 1 & 0.0490 & 6 & 1 & 0.0348 \\
\hline 20 & 1 & 0.0483 & 22 & 1 & 0.0319 \\
\hline 8 & 7 & 0.0447 & 24 & 1 & 0.0315 \\
\hline 22 & 1 & 0.0422 & 8 & 2 & 0.0277 \\
\hline 8 & 6 & 0.0420 & 25 & 1 & 0.0239 \\
\hline 24 & 1 & 0.0405 & 24 & 2 & 0.0235 \\
\hline 14 & 12 & 0.0373 & 22 & 2 & 0.0226 \\
\hline 16 & 2 & 0.0348 & 20 & 2 & 0.0201 \\
\hline 24 & 2 & 0.0345 & 8 & 7 & 0.0200 \\
\hline 20 & 2 & 0.0342 & 13 & 1 & 0.0199 \\
\hline 14 & 10 & 0.0331 & 18 & 1 & 0.0193 \\
\hline 22 & 2 & 0.0325 & 25 & 2 & 0.0185 \\
\hline 14 & 11 & 0.0320 & 16 & 2 & 0.0180 \\
\hline 8 & 5 & 0.0314 & 8 & 6 & 0.0172 \\
\hline 25 & 1 & 0.0306 & 8 & 5 & 0.0166 \\
\hline 12 & 11 & 0.0296 & 19 & 1 & 0.0156 \\
\hline 13 & 1 & 0.0287 & 23 & 1 & 0.0155 \\
\hline 14 & 9 & 0.0272 & 7 & 1 & 0.0155 \\
\hline 12 & 10 & 0.0271 & 14 & 11 & 0.0146 \\
\hline 25 & 2 & 0.0269 & 14 & 10 & 0.0136 \\
\hline
\end{tabular}

ment charge $Z_{k}$ in Fig. $3(\mathrm{a})$ and $\sqrt{\sum_{k} S_{j k}^{2}}$ as a function of the projectile charge $Z_{j}$ in Fig. 3(b). Fig. 3(a) clearly shows the dominance of fragments with $Z=1$ (protons) and $Z=2$ (alpha particles) while Fig. 3(b) shows that fragmentations of projectiles such as $\mathrm{Fe}, \mathrm{Si}, \mathrm{Mg}$ and $\mathrm{O}$ have the largest effects on the uncertainty of the dose equivalent behind shielding.

\section{BEYOND THE THIN-SHIELDING LIMIT}

Eqs. (14) and (15) derived in the thin-shielding limit can be written as

$$
\frac{d \delta H(x)}{d x} \equiv \rho \sum_{j, k} S_{j k}(x) \frac{\delta \sigma_{k j}}{\sigma_{k j}}
$$

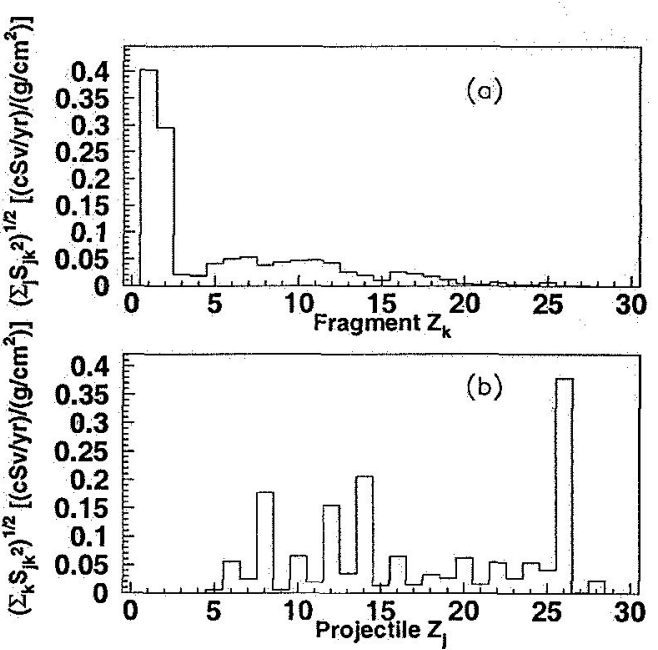

FIG. 3: Fragment (a) and projectile (b) distributions for the sensitivity of dose equivalent to a given relative change in the partial cross sections.

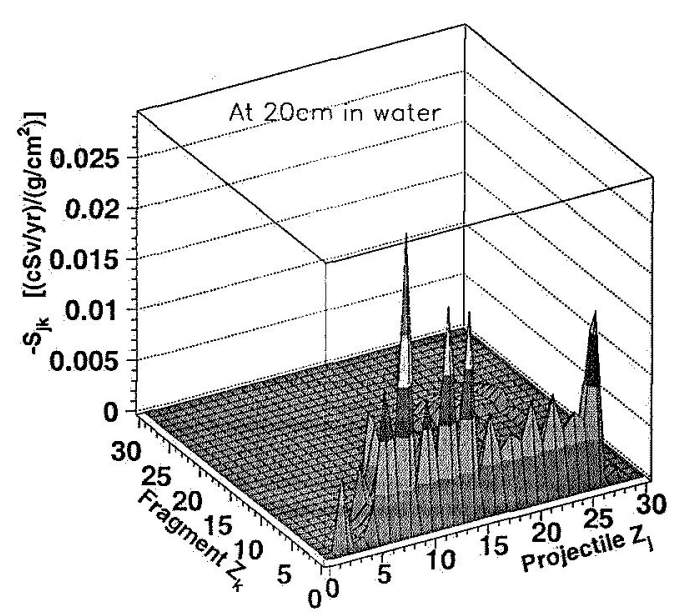

FIG. 4: (Color online) Matrix elements for the sensitivity of dose equivalent at $20 \mathrm{~g} / \mathrm{cm}^{2}$ in water shielding to a given relative change in each partial cross section.

$$
\begin{aligned}
S_{j k}(x) & =\frac{n}{\rho_{T} \rho} \int J_{j}(E, x)\left[-Z_{j}^{2} Q\left(Z_{j}^{2} L_{1}\right) \frac{A_{k}}{A_{j}}\right. \\
& \left.+Z_{k}^{2} Q\left(Z_{k}^{2} L_{1}\right)\right] L_{1} \sigma_{k j} d E
\end{aligned}
$$

where $S_{j k}(x)$ represents the sensitivity matrix element at any given depth $x$ in a shielding material. Note that Eq. (18) gives the rate of the dose equivalent change, i.e., the dose equivalent change per. unit depth, at depth $x$ from energy-independent relative changes in the partial cross sections. 


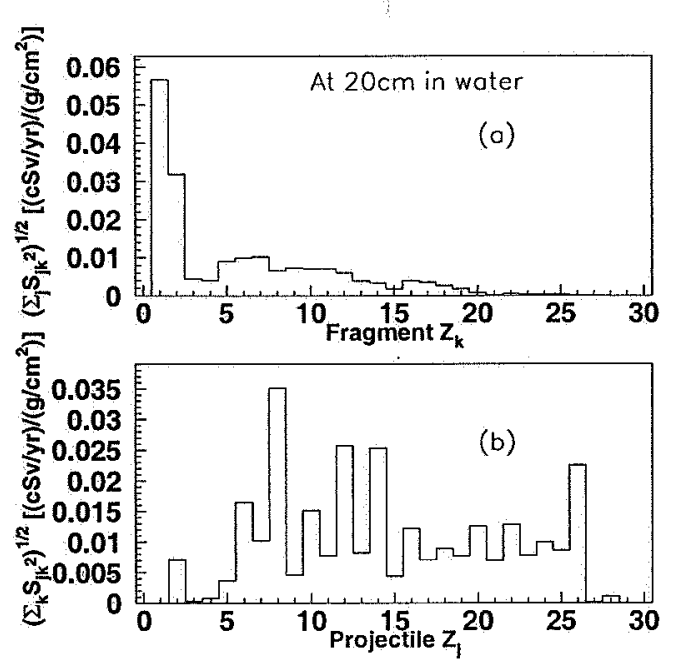

FIG. 5: Fragment (a) and projectile (b) distributions for the sensitivity of dose equivalent at $20 \mathrm{~g} / \mathrm{cm}^{2}$ in water shielding to a given relative change in the partial cross sections.

As an example, using the deterministic radiation transport code HZETRN [8] we have calculated the particle spectra at $20 \mathrm{~g} / \mathrm{cm}^{2}$ in water shielding, which were then used as the input $J_{j}$ to evaluate the sensitivity matrix elements according to Eq. (19). Fig. 4 shows the sensitivity matrix $S_{j k}$ at $20 \mathrm{~g} / \mathrm{cm}^{2}$ of water shielding, and Fig. 5 shows the one-dimensional reductions of that sensitivity matrix. First of all, compared with the results in the thin-shielding limit (i.e, at $x \sim 0 \mathrm{~g} / \mathrm{cm}^{2}$ of water shielding), we see that at $20 \mathrm{~g} / \mathrm{cm}^{2}$ of water shielding projectiles such as $\mathrm{O}$ and $\mathrm{Mg}$ are relatively more important while the Fe projectile is relatively less important. This is mainly because heavier projectiles have larger inelastic cross sections, and thus their fluences tend to decrease more inside materials. Secondly, Fig. 5(a) shows that the fragment distribution at this depth has almost the same shape as that in Fig. 3(a), therefore cross sections of light fragment productions are still the dominant source of uncertainty. Also, we see that the sensitivity matrix elements at $20 \mathrm{~g} / \mathrm{cm}^{2}$ of water shielding are much smaller in magnitudes, which reflects the overall decrease of the dose equivalent behind shielding as well as the decreased efficiency of fragmentations after a significant amount of shielding.

The total sensitivity of the dose equivalent behind a shielding material of thickness $L$ to cross sections can be evaluated according to Eq. (18) as

$$
\int_{0}^{L} \frac{d \delta H(x)}{d x} d x=\rho \sum_{j, k}\left[\int_{0}^{L} S_{j k}(x) d x\right] \frac{\delta \sigma_{k j}}{\sigma_{k j}} .
$$

In a simplified case where the errors in the partial cross sections have the same magnitude as well as the same $\operatorname{sign}\left(\delta \sigma_{k j} / \sigma_{k j} \equiv \epsilon^{\prime}\right)$, Eq. (20) gives $\rho \epsilon^{\prime} \sum_{j, k} \int_{0}^{L} S_{j k}(x) d x$.
Conversely, in the same simplified case as for Eq. (17), where the uncertainties in the partial cross sections have the same magnitude $\left(\left|\delta \sigma_{k j}\right| / \sigma_{k j} \equiv \epsilon\right)$ but random signs, Eq. (20) reduces to

$$
\int_{0}^{L} \frac{d \delta H(x)}{d x} d x \approx \rho \epsilon \sqrt{\sum_{j, k}\left[\int_{0}^{L} S_{j k}(x) d x\right]^{2}} .
$$

In any case, the sensitivity for a thick shielding is dominated by the depths at which the sensitivity matrix elements have the largest values. Fig. 6 shows the projectile distributions, represented by $\sqrt{\sum_{k} S_{j k}^{2}}$, at four different depths in water shielding, and it shows that the sensitivity matrix elements for charged fragments have larger values at smaller depths for GCR environments [10]. This suggests that the semi-analytical results in the thin-shielding limit are representative of the total sensitivity for GCR propagation through a relatively thick material.

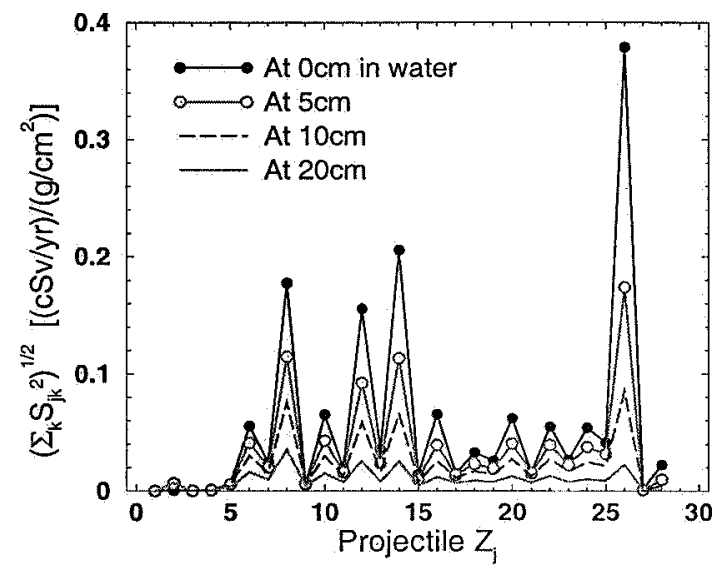

FIG. 6: (Color online) Projectile distributions for the sensitivity of dose equivalent at $0,5,10$ and $20 \mathrm{~g} / \mathrm{cm}^{2}$ in water shielding to a given relative change in the partial cross sections.

\section{DISCUSSIONS}

To illustrate the effects of the unitarity constraint of Eq. (9), we show in Fig. 7 the matrix elements without imposing the unitarity relation. In this case the terms with a negative sign in Eqs. (7), (10), (12), (15) and (19), have been neglected, leading to the following matrix elements:

$$
S_{j k}^{N}=\frac{n}{\rho_{T} \rho} \int J_{j} Z_{k}^{2} Q\left(Z_{k}^{2} L_{1}\right) L_{1} \sigma_{k j} d E .
$$

In Fig. 7 we find that heavy fragments with charges near that of the projectile are the most important for a given 
projectile; an expected feature due to the strong $Z_{k}$ dependence in $S_{j k}^{N}$. On the other hand, results with the unitarity constraint shown in Fig. 2 show totally different fragment distributions. We also see that the matrix elements without the unitarity constraint in Fig. 7 are positive, while those in Fig. 2 with the unitarity constraint are mostly negative, indicating that in most cases an independent increase of a partial cross section decreases the dose equivalent behind shielding.

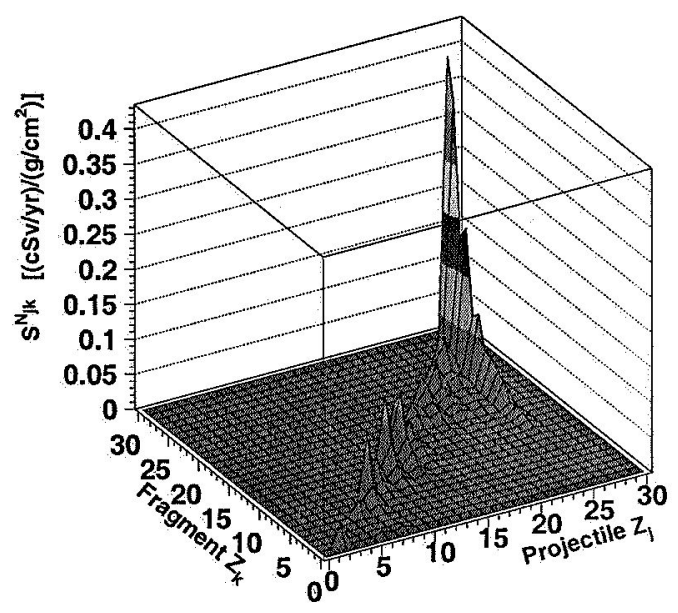

FIG. 7: (Color online) Matrix elements, without imposing the unitarity constraint, for the sensitivity of dose equivalent to a given relative change in each partial cross section.

The semi-analytical method provides the theoretical understanding of the sensitivity of the shielded dose equivalent to uncertainties in the partial cross sections. The next step is to apply the semi-analytical method in combination with our knowledge on fragmentation cross sections and on their uncertainties, where other implementations of the unitarity constraint may be needed. After a particular $\sigma_{k j}$ is changed, instead of changing the total inelastic cross section $\sigma_{j}$ to satisfy Eq. (9) a combination of the total inelastic cross section and one or more other partial cross sections may be changed. In this case, however, there is no unique definition for the sensitivity to a single partial cross section because the effect will always correspond to changes in multiple partial cross sections [11]. As our goal is to establish the priorities for partial cross sections to be further studied, how to implement the unitarity constraint should depend on our knowledge of these cross sections, especially the sizes of their errors and the correlations among them. Correlations in experimental data are certainly possible among partial cross sections, as well as between partial cross sections and the total inelastic cross section.

To explore other ways of implementing the unitarity constraint, we consider two examples where total inelastic cross sections $\sigma_{j}$ are kept the same. The first example

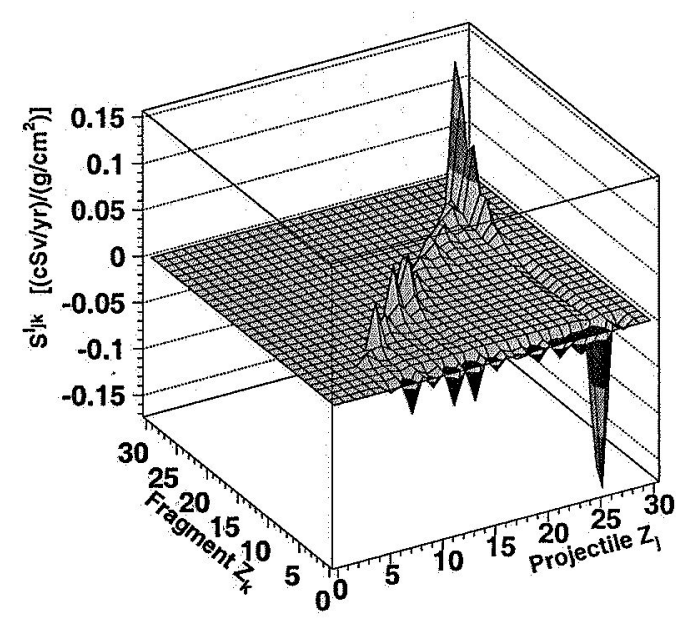

FIG. 8: (Color online) Correlated matrix elements for the sensitivity of dose equivalent to a given relative change in each partial cross section $\sigma_{k j}$, with the unitarity constraint of Eq. (9) being satisfied by fixing $\sigma_{j}$ and changing all other partial cross sections by the same fraction.

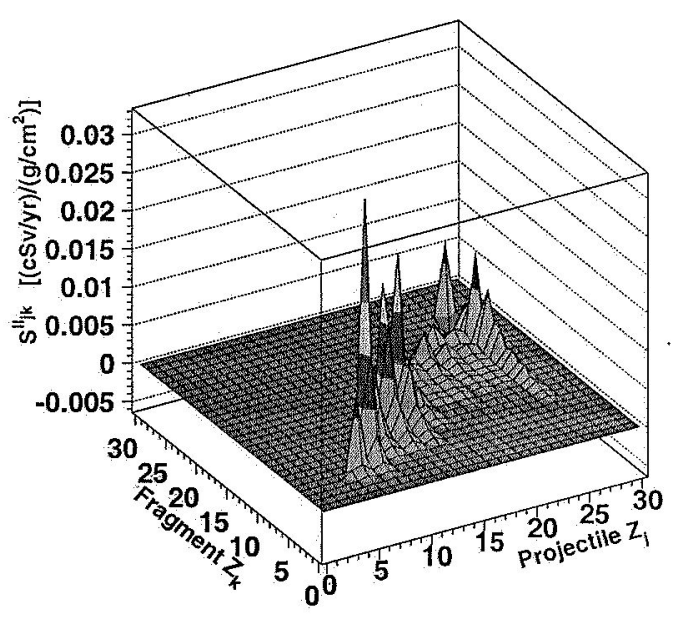

FIG. 9: (Color online) Correlated matrix elements for the sensitivity of dose equivalent to a given relative change in each partial cross section $\sigma_{k j}$, with the unitarity constraint of Eq. (9) being satisfied by fixing $\sigma_{j}$ and changing $\sigma_{k-1, j}$.

assumes that the total inelastic cross sections are known exactly but uncertainties in the partial cross sections are mostly uncorrelated. Thus a change in $\sigma_{k j}$ can be accompanied by changes in all other partial cross sections, $\sigma_{i j}(i \neq k)$, by the same fraction in order to satisfy the unitarity constraint of Eq. (9). The resulting sensitivity matrix elements are shown in Fig. 8 , and we see that 
both heavy and light fragments are important in this case. The second example also assumes that the total inelastic cross sections are known exactly but there are misidentifications of particles with charge $Z_{k}$ as particles with charge $Z_{k-1}$ (for all $k$ ), where an under-measured $\sigma_{k j}$ data would be correlated with an correspondingly over-measured $\sigma_{k-1, j}$ data. In this case a change in $\sigma_{k j}$ is accompanied by a corresponding change in $\sigma_{k-1, j}$ in order to satisfy Eq. (9). The resulting sensitivity matrix elements are shown in Fig. 9, and we see that fragments with charges near that of the projectile for certain medium-sized projectiles are the most important. This feature can be understood using the matrix elements:

$$
S_{j k}^{I I}=\frac{n}{\rho_{T} \rho} \int J_{j}\left[Z_{k}^{2} Q\left(Z_{k}^{2} L_{1}\right)-Z_{k-1}^{2} Q\left(Z_{k-1}^{2} L_{1}\right) \frac{A_{k}}{A_{k-1}}\right] L_{1} \sigma_{k j} d E,(23)
$$

which, in the simplified case where fragments have the same quality factor and the $A / Z$ ratio as those of the projectile, result in $S_{j k}^{I I} \propto S_{j k}^{N} / Z_{k}$.

We emphasize that a smaller relative uncertainty in the total inelastic cross section than in the partial cross sections does not necessarily mean that one has to keep the total inelastic cross section $\sigma_{j}$ fixed, because the smaller uncertainty may simply result from the fact that the total inelastic cross section is the sum of the partial cross sections $[5,7]$. Consider the simplified case where there are $N_{j}$ partial cross sections for projectile $j$ with similar values of $A_{k} \sigma_{k j}$ and similar magnitudes of uncertainty $\left(\left|\delta \sigma_{k j}\right| / \sigma_{k j} \equiv \epsilon\right)$ with random signs, while the other partial cross sections have negligible values of $A_{k} \sigma_{k j}$, Eq. (9) then gives

$$
\frac{\delta \sigma_{j}}{\sigma_{j}} \simeq \frac{\epsilon}{\sqrt{N_{j}}},
$$

therefore the uncertainty in the total inelastic cross section is much smaller.

To see the extent to which the sensitivity matrix depends on solar cycle variations of the GCR environments, Fig. 10 shows the one-dimensional reductions of the sensitivity matrix $S_{j k}$ in water shielding for the 1989 solar maximum GCR environment. Compared with results for the 1977 solar minimum GCR environment as shown in Fig. 3, we find that the sensitivity matrix has similar features but the overall normalization is lower mainly because of the smaller fluences of GCR particles during solar maximum.

This study addresses the important cross sections for the protection against the exposure to galactic cosmic rays. Such a study on the protection against solar particle events will be useful since they also pose a serious radiation hazard. However, we note that the straight-ahead approximation assumed in this semi-analytical method does not apply well to SEPs because of their dominant low-energy part of the spectra, and charge conservation may impose an important constraint as the majority of the nuclear fragmentation processes will occur below the pion production threshold.

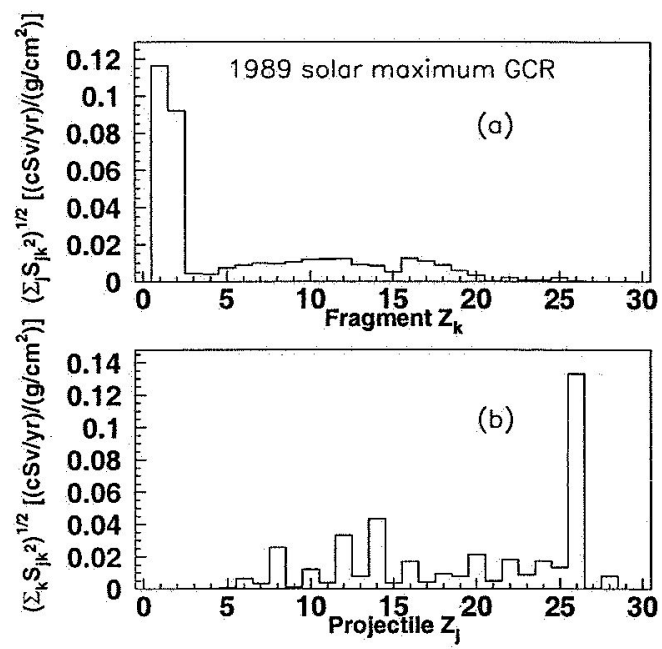

FIG. 10: Fragment (a) and projectile (b) distributions for the sensitivity of dose equivalent to a given relative change in the partial cross sections for the 1989 solar maximum GCR environment.

\section{CONCLUSION}

In this study we have developed a semi-analytical method to calculate the contribution to the uncertainties of the dose equivalent after shielding from each partial cross sections of nuclear fragmentation in the shielding materials. This method is able to point to the most important partial cross sections of nuclear fragmentations for radiation protection of astronauts against galactic cosmic rays in long-term space missions. By varying each partial cross section independently while satisfying the unitarity relation due to the baryon number conservation, we find that light fragment productions from heavy ions such as $\mathrm{Fe}, \mathrm{Si}, \mathrm{Mg}$ and $\mathrm{O}$ appear the most important. 'Thus, better theoretical and experimental understanding of these cross sections will help reduce the uncertainty in the radiation health risk predictions in long-term space missions. The unitarity relation required by baryon number conservation is shown to have significant effects on the sensitivity matrix as it correlates the uncertainties in the cross sections. At a finite depth in the shielding material, projectiles such as $\mathrm{O}$ and $\mathrm{Mg}$ become relatively more important while the $\mathrm{Fe}$ projectile becomes relatively less important, and the matrix elements have much lower magnitudes. Therefore, the thin-shielding results should be representative of the total sensitivity for thick shielding.

The next step is to apply the semi-analytical method in combination with our knowledge on fragmentation cross sections and their uncertainties. We have provided two simple examples, where different implementations of the unitarity relation may be used depending on current uncertainties in our knowledge of the fragmentation cross 
sections. We have also shown that the sensitivity matrix for a solar maximum GCR environment is similar to that for a solar minimum GCR environment except that the overall magnitude is much smaller.

\section{Acknowledgments}

The author thanks J. H. Adams, Jr. for discussions, and thanks A. F. Barghouty for discussions and careful reading of the manuscript. The author also acknowledges the support of the National Space Science \& Technology Center through cooperative agreements NCC8-200 and NNM05AA22A.
[1] F. A. Cucinotta, M.-H. Y. Kim and L. Ren, NASA Technical Paper TP-213164 PT1 (2005).

[2] See, for example, NASA's Space Radiation Project, URL at http://hacd.jsc.nasa.gov/projects/space_radiation.cfm

[3] J. R. Letaw, R. Silberberg and C. H. Tsao, ApJ Supp. 56, 369 (1984)

[4] The 1990 Recommendations of the International Commission on Radiological Protection, ICRP Publication 60, Ann. ICRP 21 (1-3) (1991).

[5] Z. W. Lin, Proceedings of the Space Nuclear Conference 2005, Paper 1093, San Diego, CA.

[6] Z. W. Lin and J. H. Adams Jr., Proceedings of the 29th International Cosmic Ray Conference (ICRC) 2, 429-432
(2005).

[7] Z. W. Lin and J. H. Adams Jr., to appear in Radiat. Res.

[8] J. W. Wilson et al., NASA Technical Paper TP-3495 (1995).

[9] J. W. Wilson et al., Nucl. Instrum. \& Methods Phys. Res. B 94, 95 (1994).

[10] The sensitivity matrix elements for neutron fragments, which could be important, have not been included in this study because the straight-ahead approximation does not apply to neutron transport in materials.

[11] J. H. Heinbockel et al., NASA Technical Paper TP213945 (2005) 